\title{
Intronic CYP46 polymorphism along with ApoE genotype in sporadic Alzheimer Disease: from risk factors to disease modulators
}

\author{
Barbara Borroni ${ }^{a}$, Silvana Archetti ${ }^{b}$, Chiara Agosti ${ }^{a}$, Nabil Akkawi ${ }^{a}$, Cristina Brambilla ${ }^{a}$, \\ Luigi Caimi $^{\mathrm{c}}$, Carlo Caltagirone ${ }^{\mathrm{d}}$, Monica Di Luca ${ }^{\mathrm{e}}$, Alessandro Padovani ${ }^{\mathrm{a}, *}$ \\ a Neurologic Clinic, University of Brescia, Spedali Civili de Brescia, Piazzale Spedali Civili 1, Brescia 25123, Italy \\ ${ }^{\mathrm{b}}$ III Laboratory of Analysis, University of Brescia, Brescia, Italy \\ ${ }^{\mathrm{c}}$ Department of Biotechnology, Biochemistry Unit, University of Brescia, Brescia, Italy \\ d Neurologic Clinic, IRCCS S. Lucia, University “Tor Vergata”, Rome, Italy \\ ${ }^{\mathrm{e}}$ Centre of Excellence for Neurodegenerative Diseases, Institute of Pharmacological Sciences, University of Milan, Milan, Italy
}

Received 17 June 2003; accepted 26 August 2003

\begin{abstract}
Increasing biological and clinical findings argue for a link between brain cholesterol turnover and Alzheimer Disease (AD), high cerebral levels of the former increasing A $\beta$ load. Cerebral cholesterol elimination involves two mechanisms dependent on Apolipoprotein E (ApoE) and cholesterol 24-hydroxylase (CYP46).

The aim of this study was to evaluate an intronic variation in CYP46 (intron 2, T $\rightarrow$ C) along with ApoE genotype as risk factors for AD and to establish the correlation between CYP46/ApoE polymorphism and disease progression. One-hundred and fifty-seven AD patients, who had been followed periodically through 1-year follow-up after enrolment, and 134 age- and gender-matched controls entered the study.

The distribution of CYP46 genotypes was significantly different in AD compared to controls $(P<0.004)$, being CYP*C allele higher in AD patients $(P<0.002)$. ApoE $\varepsilon 4$ genotype was more frequent in $\mathrm{AD}(41.4 \%)$ than in controls $(15.9 \%, P<0.0001)$. The odds ratio (OR) for $\mathrm{AD}$ risk in $\mathrm{CYP} 46 * \mathrm{C}$ carriers was 2.8 , and in $\mathrm{ApoE} \varepsilon 4$ carriers was 4.05 ; the OR for having both $\mathrm{CYP} 46^{*} \mathrm{C}$ and $\mathrm{ApoE} \varepsilon 4$ was 17.75 , demonstrating the their synergic effect on $\mathrm{AD}$ risk. In $\mathrm{AD}$ patients, $\mathrm{CYP} 46^{*} \mathrm{C}$ along with ApoE $\varepsilon 4$ genotype were associated with a higher cognitive decline at 1-year follow-up $(P<0.02)$.

These findings provide direct evidence that CYP46 and ApoE polymorphisms synergically increase the risk for AD development, and influence on the rate of cognitive decline.
\end{abstract}

(C) 2003 Elsevier Inc. All rights reserved.

Keywords: Alzheimer Disease; CYP46; ApoE; Risk factors; Rate of cognitive decline

\section{Introduction}

There is growing interest in the potential contribution of cholesterol in the pathogenesis of Alzheimer Disease (AD), several evidences highlighting a role of hypercholesterolaemia in increasing AD risk and worsening disease progression $[3,12,19]$.

In fact, pre-clinical studies have shown an association between elevated mid-life serum total cholesterol levels and late-life $\mathrm{AD}$, and a reduced incidence rate of the disease has been observed in subjects treated with statins, thus suggesting that cholesterol-lowering drugs may exert a protective role on AD onset [6-9]. Linkage between cholesterol and $\mathrm{AD}$ had also been suggested by the main recognised genetic

* Corresponding author. Tel.: +39-030-3995631; fax: +39-030-3995027.

E-mail address: padovani@med.unibs.it (A. Padovani). risk factor for sporadic late-onset $\mathrm{AD}$, i.e. Apolipoprotein $\mathrm{E}$ (ApoE), which is involved in cholesterol homeostasis [18]. In fact, a wide body of data demonstrated that ApoE $\varepsilon 4$ carriers are at greater risk of developing $\mathrm{AD}$ [22].

Further, it has been demonstrated that cholesterol affects the pathogenic mechanisms of the disease, modulating the amyloid precursor protein (APP) processing, being high cholesterol levels a factor increasing $A \beta$ biogenesis and $A \beta$ toxicity $[2,15,20,21]$.

All together literature findings claim that the excess of brain cholesterol, which is locally synthesised, needs to be eliminated. The blood-brain barrier prevents cholesterol transport from the brain to the blood, thus elimination pathways are necessary [10]. Cholesterol leaves the brain by two different mechanisms: one is ApoE-dependent, $\varepsilon 4$ allele being less effective in this process, while the second involves a recently cloned gene cholesterol $24 S$-hydroxylase (CYP46) 
$[11,24]$. CYP46 is an enzyme almost exclusively located in the brain and catalyses the conversion of excess cholesterol into $24 S$-hydroxycholesterol, which is readily secreted across the blood-brain barrier into the circulation [11]. A putative functional role of CYP46 has been postulated: an increase of 24-hydroxycholesterol in cerebrospinal fluid of $\mathrm{AD}$ patients has been shown, and different groups have analysed an intronic variation of CYP46, since genetic polymorphisms might influence functionality of the corresponding protein, but with contrasting results $[5,10,16,17]$.

These observations, arguing for a key-role of genes involved in cholesterol turnover in $\mathrm{AD}$, provide the rationale of the present study, aimed to evaluate an intronic variation in CYP46 (intron 2, $\mathrm{T} \rightarrow \mathrm{C}$ ) along with ApoE genotype, as risk factors and disease modulators in $\mathrm{AD}$.

\section{Materials and methods}

\subsection{Subjects}

Patients consecutively admitted at the "Centre of Ageing Brain and Neurodegenerative Disorders", University of Brescia, Italy, entered the study. All patients performed a somatic and neurological examination, laboratory analysis, ApoE and CYP46 genotyping, and brain imaging study (Computed Tomography or Magnetic Resonance Imaging). A wide standardised multidimensional assessment evaluating global cognitive functions and behavioural disturbances was performed in each subject.

A diagnosis of probable AD was based on National Institute of Neurological and Communicative Disorders and Stroke-Alzheimer's Disease and Related Disorders Association (NINCDS-ADRDA) criteria [13].

The following exclusionary criteria for the AD group were designed to ensure that participants had probable AD as the cause of their dementia: (a) major depressive disorder, bipolar disorder, schizophrenia, substance use disorder, or mental retardation according to criteria of the DSM-IV; (b) cerebrovascular disorders, hydrocephalus, and intra-cranial mass, documented by CT or MRI within the past 12 months; (c) abnormalities in serum folate and Vitamin B12, syphilis serology, or thyroid hormones' levels; (d) a history of traumatic brain injury or another neurologic disease (e.g. Parkinson disease, Huntington disease, seizure disorders); (e) significant medical problems (e.g. poorly controlled diabetes or hypertension; cancer within the past 5 years; clinically significant hepatic, renal, cardiac or pulmonary disorders).

Moreover, an age- and gender-matched control sample from the same Italian area from which the patients were drawn was recruited. All controls were found to be cognitively intact, following medical history and neuropsychological examination.

The study was conducted in accordance with local clinical research regulations and an informed consent was required from all subjects and caregivers when indicated.
Polymorphism analyses were performed blinded to diagnosis and genotype.

\subsection{CYP46 genotype}

Genomic DNA was isolated from whole blood samples by extraction using a salting procedure.

A 285 bp polymerase chain reaction (PCR) product containing CYP46 was amplified using specific primers (forward primer: 5'-TGA AAA CGA GTT TCC CGT CC-3'; reverse primer: 5'-GTG TGA CCA GGT AAC AGT CA-3'). PCR was performed using $100 \mathrm{ng}$ of genomic DNA in $50 \mathrm{ml}$ of reaction mixture.

After initial denaturation at $95^{\circ} \mathrm{C}$ for $5 \mathrm{~min}$, reaction mixture was subjected to 32 cycles of $30 \mathrm{~s}$ denaturation at $95^{\circ} \mathrm{C}$, $30 \mathrm{~s}$ annealing at $53^{\circ} \mathrm{C}$, and $30 \mathrm{~s}$ extension at $72^{\circ} \mathrm{C}$, followed by $10 \mathrm{~min}$ extension step at $72^{\circ} \mathrm{C}$. PCR products were digested by $M s p I$ restriction enzyme. CYP46 T allele corresponded to the uncut $285 \mathrm{bp}$ fragment, while CYP46 C allele was characterised by two fragments of 209 and $76 \mathrm{bp}$.

The results of amplification and the digestion fragments were revealed by 2 and 3\% agarose gels with ethidium bromide, respectively.

\subsection{ApoE genotyping}

Genetic variation at the ApoE locus is determined by restriction isotyping using PCR amplification and subsequent digestion with HhaI (New England Biolabs). The nucleotide substitutions that result in Arg-Cys interchange at position 112 and 158 alter HhaI cleavage sites: each genotype can be distinguished by unique combinations of $\mathrm{HhaI}$ fragment sizes in all homozygotic and heterozygotic combinations.

\subsection{Study design}

$\mathrm{AD}$ patients and healthy controls were evaluated at baseline and a blood venipuncture for DNA extraction and polymorphisms' analysis was performed. In regard to $\mathrm{AD}$ patients, current available therapy with Cholinesterase Inhibitors (ChEIs) had been administered at recommended dosage, and they had been followed periodically, and 1-month and 1-year follow-up neuropsychological assessment was recorded.

\subsection{Statistical methods}

Genotype and allele frequencies between $\mathrm{AD}$ patients and control subjects were compared by Pearson $\chi^{2}$ test. Hardy-Weinberg equilibrium of the examined population was confirmed by $\chi^{2}$ test.

For all the continuous variables, the analysis of variations between groups was performed using the unadjusted ANOVA. In order to account for possible confounds (gender, age), the ANCOVA has been performed as well. Fur- 
Table 1

CYP46 and ApoE in AD patients and in controls

\begin{tabular}{lccc}
\hline & $\begin{array}{l}\text { Controls } \\
(n=134)^{\mathrm{a}}\end{array}$ & $\begin{array}{l}\mathrm{AD} \\
(n=157)^{\mathrm{a}}\end{array}$ & $P^{\mathrm{b}}$ \\
\hline CYP46 (intron 2, T $\rightarrow$ C) $\begin{array}{c}\text { genotype } \%(n) \\
\text { TT }\end{array}$ & $64.2(86)$ & $45.4(65)$ & \\
TC & $28.3(38)$ & $47.6(68)$ & \\
CC & $7.5(10)$ & $7.0(10)$ & 0.004 \\
TT & $64.2(86)$ & $45.4(65)$ & \\
CT or CC & $35.8(48)$ & $54.6(78)$ & $<0.0001$ \\
ApoE genotype \% $(n)$ & & & \\
$\varepsilon 4$ & $15.8(20)$ & $41.4(65)$ & \\
Non- $\varepsilon 4$ & $84.2(107)$ & $58.6(92)$ & $<0.0001$ \\
$\varepsilon 2$ allele frequency & $3.6(9)$ & $2.5(8)$ & \\
$\varepsilon 3$ allele frequency & $88.5(225)$ & $74.8(235)$ & \\
$\varepsilon 4$ allele frequency & $7.9(20)$ & $22.6(71)$ & $<0.0001$ \\
\hline \multicolumn{2}{c}{ a Difference on the totals are due to missing data. } \\
b Pearson $\chi^{2}$ test. & &
\end{tabular}

ther analysis was carried out by using ANOVA for repeated measures. Statistical significance was assumed at $P<0.05$.

The analysis was performed using the software STATA ${ }^{\circledR}$ 7.0 (Intercooled Stata 7.0 for Windows, Stata Corporation, College Station, TX).

\section{Results}

One hundred and fifty-seven demented patients (female: 61.8\%; age: $72.2 \pm 7.9$ ), and 134 age- and gender-matched control subjects entered the study.

The distribution of CYP46 genotypes was significantly different in $\mathrm{AD}$ compared to controls $(P=0.004$; see Table 1$)$. The presence of at least one of ${ }^{*} \mathrm{C}$ allele $\left(\mathrm{CYP} 46^{*} \mathrm{C}\right.$ : CYP46 C/T or CYP C/C) was higher in AD patients (54.6\%) compared to control subjects $(35.8 \%, P<0.0001)$.

The distribution of ApoE isoforms significantly differed between $\mathrm{AD}$ cases and controls, being $\mathrm{ApoE} \varepsilon 4$ genotype more frequent in patients (AD versus controls: $41.4 \%$ versus $15.8 \%, P<0.0001)$. The presence of at least one ApoE $\varepsilon 4$ allele (ApoE*4) was significantly associated with $\mathrm{AD}$ (AD versus controls: $22.6 \%$ versus $7.9 \%, P<0.0001$; see Table 1).

Crude OR for the risk of $\mathrm{AD}$ in $\mathrm{CYP} 46^{*} \mathrm{C}$ carriers was 2.56 (95\% confidence interval: 1.58 to 4.08 ), while for ApoE*4 carriers it was 3.78 (95\% CI: 2.13 to 6.71). Compared to subjects with neither CYP46*C nor ApoE*4, the OR for the presence of CYP46* $\mathrm{C}$ without $\mathrm{ApoE}^{*} 4$ allele was 2.81 (95\% CI: 1.60 to 4.97), the OR for the presence of ApoE*4 allele without CYP46*C was 4.05 (95\% CI: 1.95 to 8.39); the OR for having both $\mathrm{CYP}_{4}{ }^{*} \mathrm{C}$ and $\mathrm{ApoE}^{*} 4$ was 17.75 (95\% CI: 5.83 to 54.06), demonstrating their synergic effect on AD risk.

Eighty-eight patients completed the 1-year follow-up and were re-evaluated. Only patients who have never experienced ChEIs treatment before enrolment were considered at follow-up, to avoid confounds. These patients were grouped according to ApoE and CYP46 genotype. As shown in Table 2, ApoE*4/CYP46*C grouped patients, who completed 1-year follow-up, did not differ for demographic or clinical characteristics.

Rate of cognitive decline was calculated as $\triangle$ MMSE (MMSE 1 year - MMSE baseline), and factors related to $\triangle$ MMSE analysed. Adjusting for age and gender, nor ApoE*4 genotype, nor CYP $46^{*} \mathrm{C}$ genotype, but the combination ApoE $\varepsilon 4 / \mathrm{CYP} 46^{*} \mathrm{C}$ predicted $\triangle \mathrm{MMSE}(P=$ $0.0454)$. In fact, at 1-year follow-up AD patients carrying both ApoE $\varepsilon 4$ and $\mathrm{CYP}^{*} \mathrm{C}$ polymorphisms showed a higher rate of cognitive decline compared to the others $(F[2,168]=2.62, P<0.02)$, while no differences among groups were found at 1-month follow-up (see Table 2, and Fig. 1).

\section{Discussion}

It is well established that sporadic late-onset $\mathrm{AD}$ is a polygenic disease, several genetic polymorphisms being suggested as modulators of AD susceptibility [4]. Among others, several proposed genes encode for protein that are

Table 2

Demographic and clinical characteristics of AD patients who completed 1-year follow-up according to CYP46 and ApoE genotype

\begin{tabular}{|c|c|c|c|c|c|}
\hline Variable & CYP46*non-C ApoE*non-4 & CYP46*non-C ApoE*4 & CYP46*C ApoE*non-4 & CYP46*C ApoE*4 & $P^{\mathrm{b}}$ \\
\hline$n$ & 21 & 21 & 32 & 18 & - \\
\hline Age (year) & $72.3 \pm 7.7$ & $73.2 \pm 5.5$ & $69.8 \pm 10.5$ & $72.5 \pm 5.5$ & 0.45 \\
\hline Gender, F/M & $11 / 10$ & $14 / 7$ & $18 / 15$ & $13 / 5$ & $0.50^{\mathrm{c}}$ \\
\hline MMSE baseline & $20.1 \pm 5.8$ & $19.7 \pm 6.0$ & $19.3 \pm 6.3$ & $20.4 \pm 5.1$ & 0.94 \\
\hline MMSE 1-month & $20.6 \pm 5.8$ & $19.9 \pm 5.8$ & $19.7 \pm 6.0$ & $20.8 \pm 5.0$ & 0.96 \\
\hline MMSE 1-year & $19.8 \pm 5.6$ & $18.9 \pm 6.4$ & $18.5 \pm 6.5$ & $17.9 \pm 6.4$ & 0.84 \\
\hline$\triangle \mathrm{MMSE}$ & $-0.33 \pm 2.6$ & $-0.80 \pm 2.2$ & $-0.81 \pm 2.3$ & $-2.5 \pm 3.3$ & 0.06 \\
\hline
\end{tabular}

MMSE: Mini-Mental State Examination; $\triangle$ MMSE: MMSE 1-year - MMSE baseline CYP46*non-C: CYP46 TT; CYP46*C: CYP46 TC or CYP46 CC; ApoE*non-4: ApoE $\varepsilon 2 / \varepsilon 2$ or ApoE $\varepsilon 3 / \varepsilon 3$ or ApoE $\varepsilon 3 / \varepsilon 2$ or ApoE $\varepsilon 2 / \varepsilon 2$; ApoE*4: ApoE $\varepsilon 3 / \varepsilon 4$ or ApoE $\varepsilon 4 / \varepsilon 4$.

${ }^{\mathrm{a}}$ Mean ( \pm S.D.).

${ }^{\mathrm{b}}$ Unadjusted ANOVA.

${ }^{\mathrm{c}}$ Pearson $\chi^{2}$ test. 


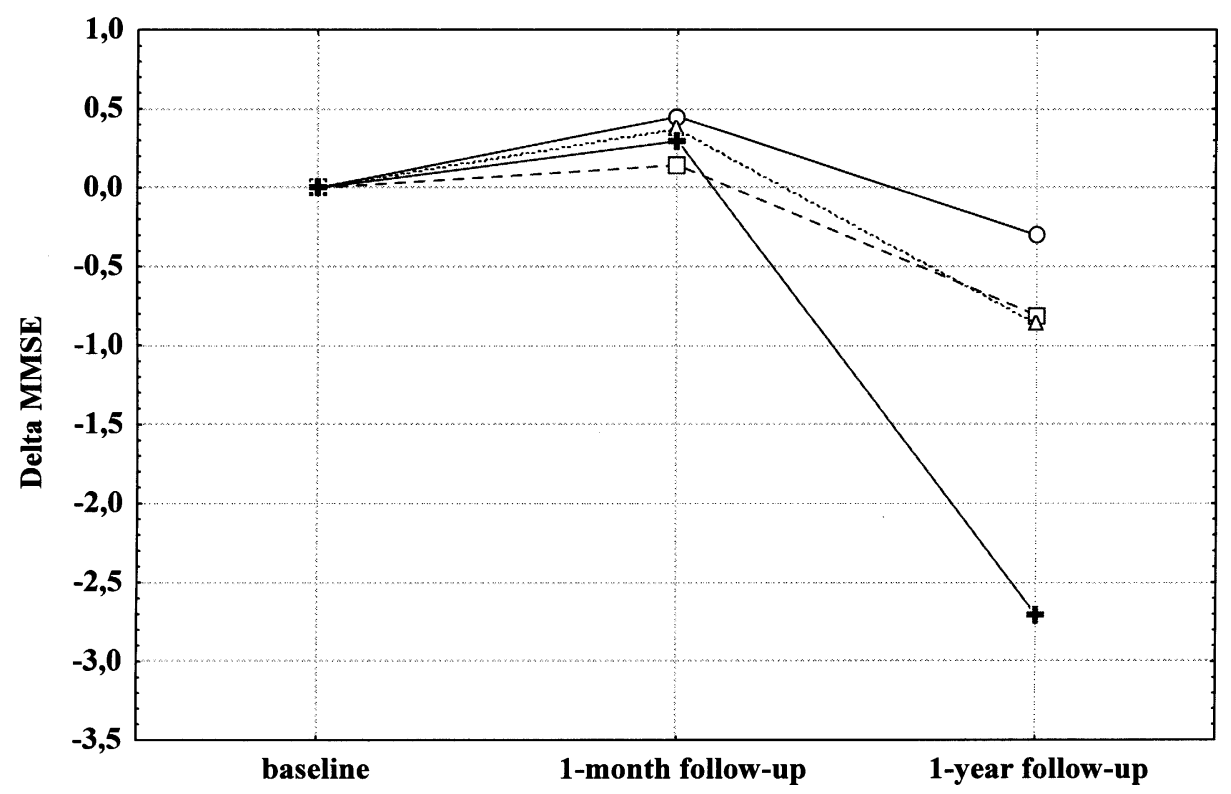

Fig. 1. Changes in MMSE scores at 1-month and 1-year follow-up in AD patients according to CYP46 and ApoE polymorphisms. - - , ApoE*4/CYP46*C; $\cdots \bullet \cdots$, ApoE*non-4/CYP46*C; $\cdots \square \cdots \cdot$, ApoE*4/CYP*non-C; - - , ApoE*non-4/CYP*non-C. $\Delta$ MMSE: Mini-Mental State Examination

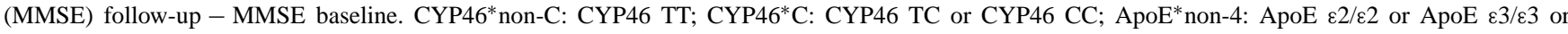
ApoE $\varepsilon 3 / \varepsilon 2$; or ApoE*4: ApoE $\varepsilon 3 / \varepsilon 4$ or ApoE $\varepsilon 4 / \varepsilon 4$.

involved in lipid homeostasis, thus claiming that cholesterol plays a key-role in AD pathogenesis [1].

In this study, we reported that an intronic variation in CYP46, CYP $46^{*} \mathrm{C}$, along with ApoE $\varepsilon 4$ genotype sinergically increase the risk of $\mathrm{AD}$ development; further, $\mathrm{AD}$ patients carrying the combination of these two polymorphisms showed a higher rate of cognitive decline at 1-year follow-up.

CYP46 and ApoE are involved in the pathways by which excess brain cholesterol is transported into circulation [10]. Biological evidences have strengthened the role of these two proteins in AD. In fact, it is well established that ApoE induces cholesterol efflux in an isoform-dependent manner, as the most recognised sporadic AD-related risk factor $\varepsilon 4$ allele appears to be less effective in this process [14]. Further, it has been demonstrated that AD is characterised by abnormal induction of CYP46 enzyme, which leads to changes in 24S-hydroxycholesterol from brain to circulation [17,23]. Neuropathological evidences reported that high concentration of CYP46 has been found in glial cells in AD brains, suggesting that an imbalance of cholesterol turnover may be a feature of reactive astroglia in the disease [4].

How CYP46 polymorphisms, such as intronic variation here reported, affect protein function has not been established yet. It could be speculated that intronic variations can either influence on the rate of transcription of gene products by affecting splice sites or modulate nuclear trascriptional factors.

According to this, an imbalance of cholesterol turnover, due to less effective physiological mechanisms, such as that determined by ApoE or CYP46 genetic variations, could represent a risk factor for $\mathrm{AD}$ development, and could worsen disease progression once symptomathology is overt. In agreement, $\mathrm{AD}$ patients carrying both $\mathrm{CYP} 46^{*} \mathrm{C}$ and $\mathrm{ApoE}^{*} 4$ progressed much more faster at 1 -year evaluation.

We acknowledge that our study has some limitations. First of all, a wider sample of subjects is needed as well as hypothesis-confirming sample from other countries. Secondly, further biological studies analysing CYP46 polymorphisms's effect on cholesterol metabolism are mandatory.

Despite these limitations, this study underlines once more the importance of taking into account the enzymatic pathways which are involved in cholesterol homeostasis to better understand how they influence on AD development. The complex interplay between genetic and environmental factors should be introduced into future trials to fully appreciate individual susceptibility and prognosis' determinants in $\mathrm{AD}$. In fact, the present findings argue for a double implication of CYP46 and ApoE genotyping, since these genes act both as risk factors and disease modulators in AD.

Establishing the real impact of the intricate maze of these genetic polymorphisms and corresponding proteins' function represents a key-issue in the progress of the pathology's knowledge, leading to a better definition of the disease and providing many potential opportunities to design preventive therapeutic strategies.

\section{Acknowledgments}

The authors wish to thank Michela Cossandi for excellent technical assistance, Dr. Cristian Pattaro for valuable 
statistical analysis, and Dr. Elisabetta Cottini for critical review of the manuscript.

\section{References}

[1] Bogdanovic N, Bretillon L, Lund EG, Diczfalusy U, Lannfelt L, Winblad B, et al. On the turnover of brain cholesterol in patients with Alzheimer's disease. Abnormal induction of the cholesterol-catabolic enzyme CYP46 in glial cells. Neurosci Lett 2001;314:45-8.

[2] Borroni B, Colciaghi F, Lenzi GL, Caimi L, Cattabeni F, Di Luca M, Padovani A. High cholesterol affects platelet APP processing in controls and in AD patients. Neurobiol Aging 2003;24(5):631-6.

[3] Borroni B, Pettenati C, Bordonali T, Akkawi N, Di Luca M, Padovani A. Serum cholesterol levels modulate long-term efficacy of cholinesterase inhibitors in Alzheimer Disease. Neurosci Lett 2003;343:213-5.

[4] Chapman PF, Falinska AM, Knevett SG, Ramsay MF. Genes, models and Alzheimer's disease. Trends Genet 2001;17:254-61.

[5] Desai P, DeKosky ST, Kamboh MI. Genetic variation in the cholesterol 24-hydroxylase (CYP46) gene and the risk of Alzheimer's disease. Neurosci Lett 2002;328:9-12.

[6] Fassbender K, Simons M, Bergmann C, Stroick M, Lutjohann D, Keller P, et al. Simvastatin strongly reduces levels of Alzheimer's disease beta-amyloid peptides Abeta 42 and Abeta 40 in vitro and in vivo. Proc Natl Acad Sci USA 2001;98:5856-61.

[7] Fassbender K, Stroick M, Bertsch T, Ragoschke A, Kuehl S, Walter S, et al. Effects of statins on human cerebral cholesterol metabolism and secretion of Alzheimer amyloid peptide. Neurology 2002;59:1257-8.

[8] Jick H, Zornberg GL, Jick SS, Seshadri S, Drachman DA. Statins and the risk of dementia. Lancet 2000;356:1627-31.

[9] Kivipelto M, Helkala EL, Laakso MP, Hanninen T, Hallikainen M, Alhainen K, et al. Apolipoprotein E epsilon 4 allele, elevated midlife total cholesterol level, and high midlife systolic blood pressure are independent risk factors for late-life Alzheimer disease. Ann Intern Med 2002;137:149-55.

[10] Kolsch H, Lutjohann D, Ludwig M, Schulte A, Ptok U, Jessen F, et al. Polymorphism in the cholesterol $24 S$-hydroxylase gene is associated with Alzheimer's disease. Mol Psychiatry 2002;7:899-902.

[11] Lund EG, Guileyardo JM, Russell DW. cDNA cloning of cholesterol 24-hydroxylase, a mediator of cholesterol homeostasis in the brain. Proc Natl Acad Sci USA 1999;13:7238-43.
[12] Marx J. Alzheimer's disease. Bad for heart, bad for the mind? Science $2001 ; 294: 508-9$.

[13] McKhann G, Drachman D, Folstein M, Katzman R, Price D, Stadlan EM. Clinical diagnosis of Alzheimer's disease: report of the NINCDS-ADRDA Work Group under the auspices of Department of Health and Human Services Task Force on Alzheimer's Disease. Neurology 1984;34:939-44.

[14] Michikawa M, Fan QW, Isobe I, Yanagisawa K. Apolipoprotein E exhibits isoform-specific promotion of lipid efflux from astrocytes and neurons in culture. J Neurochem 2000;74:1008-16.

[15] Michikawa M. The role of cholesterol in pathogenesis of Alzheimer's disease: dual metabolic interaction between amyloid beta-protein and cholesterol. Mol Neurobiol 2003;27:1-12.

[16] Papassotiropoulos A, Lutjohann D, Bagli M, Locatelli S, Jessen F, Buschfort R, et al. 24S-hydroxycholesterol in cerebrospinal fluid is elevated in early stages of dementia. J Psychiatr Res 2002;36:27-32.

[17] Papassotiropoulos A, Streffer JR, Tsolaki M, Schmid S, Thal D, Nicosia F, et al. Increased brain beta-amyloid load, phosphorylated tau, and risk of Alzheimer disease associated with an intronic CYP46 polymorphism. Arch Neurol 2003;60:29-35.

[18] Poirier J. Apolipoprotein E and cholesterol metabolism in the pathogenesis and treatment of Alzheimer's disease. Trends Mol Med 2003;9:94-101.

[19] Puglielli L, Tanzi RE, Kovacs DM. Alzheimer's disease: the cholesterol connection. Nat Neurosci 2003;6:345-51.

[20] Refolo LM, Malester B, LaFrancois J, Bryant-Thomas T, Wang R, Tint GS, et al. Hypercholesterolemia accelerates the Alzheimer's amyloid pathology in a transgenic mouse model. Neurobiol Dis 2000;7:321-31.

[21] Refolo LM, Pappolla MA, LaFrancois J, Malester B, Schmidt $\mathrm{SD}$, Thomas-Bryant $\mathrm{T}$, et al. A cholesterol-lowering drug reduces beta-amyloid pathology in a transgenic mouse model of Alzheimer's disease. Neurobiol Dis 2001;8:890-9.

[22] Saunders AM, Strittmatter WJ, Schmechel D, George-Hyslop PH, Pericak-Vance MA, Joo SH, et al. Association of apolipoprotein E allele epsilon 4 with late-onset familial and sporadic Alzheimer's disease. Neurology 1993;43:1467-72.

[23] Vega GL, Weiner MF, Lipton AM, Von Bergmann K, Lutjohann $\mathrm{D}$, Moore C, et al. Reduction in levels of $24 S$-hydroxycholesterol by statin treatment in patients with Alzheimer disease. Arch Neurol 2003;60:510-5.

[24] Wolozin B. CYP46 (24-cholesterol hydroxylase). A genetic risk factor for Alzheimer disease. Arch Neurol 2003;60:16-8. 